\title{
EVALUACIÓN GEOQUÍMICA DE RESIDUOS MINEROS (JALES O COLAS) DE MINERALIZACIÓN DE TIPO EPITERMAL, HIDALGO, MÉXICO
}

\author{
GEOCHEMICAL EVALUATION OF MINING WASTES (TAILINGS) OF \\ EPITHERMAL ORE MINERALIZATION, HIDALGO, MEXICO
}

\author{
Raúl Moreno $^{1 \& 2 *}$, Marcos G. Monroy², E. Pedro Castañeda ${ }^{1}$ \\ ${ }^{1}$ Área Académica de Ciencias de la Tierra y Materiales, Universidad Autónoma \\ del Estado de Hidalgo. Código postal 42184 \\ ${ }^{2}$ Instituto de Metalurgia, Universidad Autónoma de San Luis Potosí. Código \\ postal 78140, San Luis Potosí, S.L.P., México. \\ *Autor para contacto: morenot@uaeh.reduaeh.mx
}

(Recibido:12/03/09 ; aceptado: 12/12/09)

\begin{abstract}
The mining district Pachuca-Real del Monte is located to the center-east of Mexico, between the geographical coordinates $20^{\circ} 07^{\prime} 30^{\prime}$ " of north latitude and $98^{\circ} 44^{\prime} 00^{\prime \prime}$ of length west. The residues are derived from an epithermal mineralization. Concentrations of $\mathrm{Pb}$ and the $\mathrm{Mn}$ are significant. The mineralization consists of pyrite, limonite-goethite-hematite, sphalerite, galena, and chalcopyrite associated with quartz, calcite and silicates. X-rays diffraction patterns determined quartz as the mayor mineral phase, with minor gypsum, calcite, alunite, and pyrite. The statistical analysis showed the frequent association of $\mathrm{Zn}-\mathrm{Cd}$. The microanalysis with the scanning electronic microscope and electronic microprobe showed the neoformation of the pyrite and the oxidation and precipitate of sulfides. The tests NOM-053 and EPA-1312 showed that the Cd, Ni, Ag, Pb Mn, Zn, and As do not exceed the LMP. Only the Pb is superior to the LMP in the first test. These residues might be classified as dangerous with regard to the $\mathrm{Pb}$.

Key words: Mining district, México, epithermal, tailings, neoformation
\end{abstract}

RESUMEN: El distrito minero Pachuca-Real del Monte se localiza al centro-oriente de México, entre las coordenadas geográficas $20^{\circ} 07^{\prime} 30^{\prime \prime}$ de latitud norte y $98^{\circ} 44^{\prime} 00^{\prime}$ ' de longitud oeste. Los residuos son derivados de una mineralización epitermal. Las concentraciones de $\mathrm{Pb}$ y el Mn son significativas. La mineralización está constituida por pirita, limonita-goethita-hematita, esfalerita, galena y calcopirita asociada a cuarzo, calcita y silicatos. La difracción de rayos$\mathrm{X}$ determinó el cuarzo como fase mineral mayoritaria y al yeso, calcita, alunita y pirita como minoritarias. El análisis estadístico mostró la frecuente asociación Zn-Cd. El microanálisis del microscopio electrónico de barrido y microsonda electrónica mostró la neoformación de la pirita y la oxidación y precipitación de sulfuros. Las pruebas NOM-053 y EPA-1312 mostraron que el Cd, Ni, Ag, Pb Mn, Zn y As no rebasan el LMP. Solo el Pb es superior al LMP en la primera prueba. Estos residuos podrían ser clasificados como peligrosos con respecto al $\mathrm{Pb}$.

Palabras clave: Distrito minero, México, epitermal, jales (colas), neoformación.

MORENO, R., MONROY, M.G. \& CASTANEDA, E.P., 2009: Geochemical evaluation of mining wastes (tailings) of epithermal ore mineralization, Hidalgo, Mexico.- Rev. Geól. Amér. Central, 41: 79-98. 


\section{INTRODUCCIÓN}

En México, la minería es una de las actividades económicas de mayor tradición, la cual se ha desarrollado por casi cinco siglos. Es importante mencionar que esta actividad se ha beneficiado con la evolución tecnológica en el beneficio y la recuperación de metales, desde los procesos primitivos de beneficio por amalgamación, cianuración, flotación, hasta los procesos modernos de lixiviación y fundición. Sin embargo, esta actividad también conlleva un problema de actualidad, la acumulación de residuos mineros (jales y terreros) que se ha producido durante este período (Moreno, 1998). El manejo inadecuado y la disposición de estos residuos, ha generado problemas de tipo ambiental en todo el mundo. Entre los cuales, por su importancia se pueden mencionar los siguientes: (1) - la generación de drenaje ácido de roca (DAR) acompañado de la lixiviación de metales (LM) y metaloides (Freeze \& Cherry, 1979; Blowes \& Ptacek, 1994); (2) - la falla en estructuras de retención (diques) de los depósitos de los jales (presas y/o embalses), ocasionando por su naturaleza (tamaño de partícula, plasticidad y contenido de agua) avalanchas que se desplazan a las partes más bajas de valles y algunas veces se depositan en los cauces de los ríos (Kelly \& Spottiswod, 1990); (3) - la dispersión eólica asociada al tamaño de partícula, siendo el mineral muy fino fácilmente transportado por el viento, siempre y cuando el depósito no alcance una cierta consolidación o el jal sea removido de su depósito original (Caldwell \& Welsh, 1982); y (4) - la contaminación visual que se aprecia en las zonas que en la actualidad han sido urbanizadas, dando un aspecto deprimente debido a la ausencia de medidas de restauración de estos depósitos (Caldwell \& Welsh, 1982).

En México, los yacimientos económicos se encuentran distribuidos a través del país bajo un patrón de franjas metálicas de dirección NW-SE subparalelas a la paleotrinchera de México. Estos yacimientos están relacionados al magmatismo tardi-laramídico (intrusiones): los de tipo pórfido de paragénesis $\mathrm{Cu}-\mathrm{Mo} \mathrm{y} \mathrm{los} \mathrm{de} \mathrm{tipo} \mathrm{skarn} \mathrm{de} \mathrm{para-}$ génesis $\mathrm{Zn}-\mathrm{Pb}-\mathrm{Ag}(\mathrm{Cu})$; y los epitermales relacionados a un vulcanismo terciario post-laramídico (etapa de cuencas y sierras) de paragénesis $\mathrm{Pb}$ Zn-Ag y Au-Ag (Damon, et al., 1981; Yta, 1992). De manera general, los yacimientos comprendidos en estas franjas han generado un gran volumen de residuos (jales). Para este estudio se seleccionaron los jales producidos por la explotación y beneficio de uno de los yacimientos más comunes en el país, el de tipo epitermal de metales preciosos bajo en sulfuros, de paragénesis $\mathrm{Ag}-\mathrm{Au}(\mathrm{Pb}-$ $\mathrm{Zn)}$ del distrito minero Pachuca - Real del Monte. En este distrito existen cuatro presas de jales, las cuales están expuestos a las condiciones climáticas ambientales del sitio: Bajo estas condiciones ocurre la dispersión de contaminantes por medios físicos (erosión, transporte eólico y pluvial) o por su alteración y movilidad química (disolución y transporte en medio acuoso). La movilidad de los elementos potencialmente tóxicos (EPTs) es producida por medio de los procesos fisico-químicos incluyendo la interacción con el medio ambiente superficial o cercano a la superficie y la capacidad de movimiento dentro de los fluidos después de su disolución (Smith \& Huyck, 1999).

\section{METODOLOGÍA}

\section{Área de estudio}

El distrito minero Pachuca - Real del Monte se localiza en la porción centro-oriental de México, situado en la parte centro-meridional del estado de Hidalgo. Abarca una superficie de $130 \mathrm{~km}^{2}$, entre las coordenadas geográficas $20^{\circ} 07^{\prime} 30^{\prime \prime}$ de latitud norte y $98^{\circ} 44^{\prime} 00^{\prime \prime}$ de longitud oeste (Fig. 1). Está situado en una zona de clima templado que presenta una temperatura promedio de $17^{\circ} \mathrm{C}$ y una precipitación media anual de $514 \mathrm{~mm}$. El descubrimiento del distrito data del año 1552, su exploración y explotación intensiva y sistemática se llevo a cabo sólo a partir de principios del siglo veinte. Durante 35 años hasta el año 1963, la extracción del mineral fue 3000 a 4000 toneladas diarias. En el distrito afloran cuatro unidades litológicas que están constituidas de la base a la cima por un conglomerado epicontinental; un paquete volcánico andesítico; un paquete volcánico riolítico y un paquete volcánico basáltico (Yta \& 


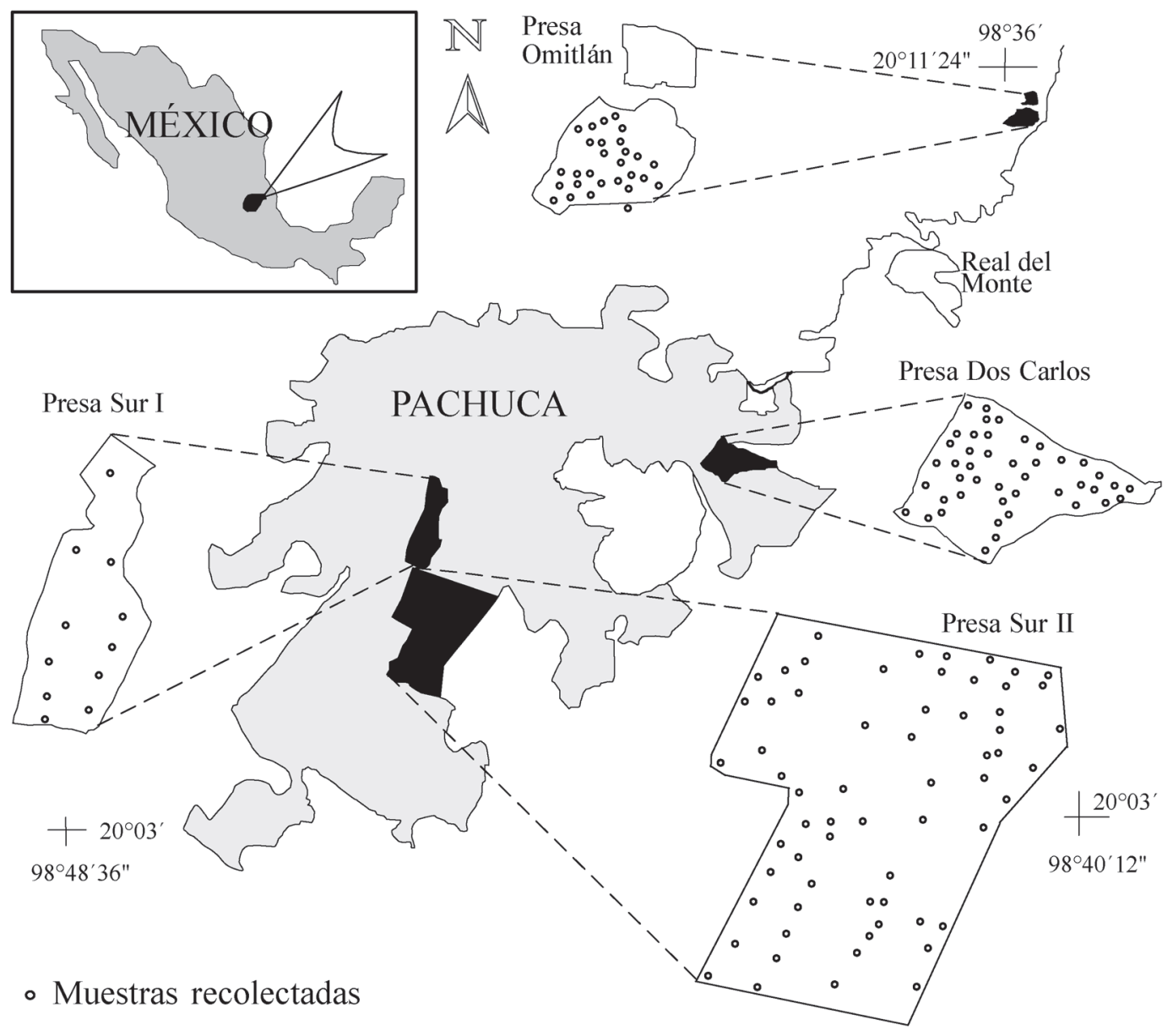

Fig. 1: Localización en el distrito minero Pachuca - Real del Monte, las presas de jales Omitlán, Dos Carlos, Sur I y Sur II, estado de Hidalgo.

Moreno, 1997). La morfología de las estructuras mineralizadas es tabular y varía tanto en espesor (cm hasta $40 \mathrm{~m}$ ), como en longitud (100 m hasta $15 \mathrm{~km}$ ). Se han definido dos sistemas de vetas E-W y N-S (Geyne et al., 1963). La mineralización está constituida por sulfuros: pirita $\left(\mathrm{FeS}_{2}\right)$, esfalerita $(\mathrm{ZnS})$, galena $(\mathrm{PbS})$, calcopirita $\left(\mathrm{CuFeS}_{2}\right)$, calcocita y covelita $\left(\mathrm{Cu}_{2} \mathrm{~S}, \mathrm{CuS}\right)$, argentita y acantita $\left(\mathrm{Ag}_{2} \mathrm{~S}\right)$ y sulfosales: polibasita $\left(9 \mathrm{Ag}_{2} \mathrm{~S} \cdot \mathrm{Sb} \cdot \mathrm{S}_{3}\right)$, estefanita $\left(5 \mathrm{Ag}_{2} \mathrm{~S} \cdot \mathrm{Sb}_{2} \mathrm{~S}\right)$, miargirita $\left(\mathrm{Ag}_{2} \mathrm{~S} \cdot \mathrm{Sb}_{2} \mathrm{~S}_{3}\right)$, pirargirita $\left(3 \mathrm{Ag}_{2} \mathrm{~S} \cdot \mathrm{Sb}_{2} \mathrm{~S}_{3}\right)$, proustita $\left(3 \mathrm{Ag}_{2} \mathrm{~S}\right.$. $\left.\mathrm{As}_{2} \mathrm{~S}_{3}\right)$ y stenbergita $\left(\mathrm{Ag}_{2} \mathrm{~S} \cdot \mathrm{Fe}_{4} \mathrm{~S}_{5}\right)$. El oro nativo se presenta escaso y en granulometría muy fina. El cuarzo $\left(\mathrm{SiO}_{2}\right)$ es el mineral más abundante en la ganga, seguido de calcita $\left(\mathrm{CaCO}_{3}\right)$ y albi- ta $\left(\mathrm{NaAlSi}_{3} \mathrm{O}_{8}\right)$. La rodonita $\left(\mathrm{MnSiO}_{3}\right)$ y la bustamita $\left[\mathrm{CaMn}_{5}\left(\mathrm{SiO}_{3}\right)_{6}\right]$ se hallan en cantidades pequeñas y normalmente se encuentran asociadas con menas ricas en plata. La clorita $\left[\mathrm{Mg}_{3}\left(\mathrm{Si}_{4} \mathrm{O}_{10}\right)\right.$ $\left.(\mathrm{OH})_{2} \mathrm{Mg}_{3}(\mathrm{OH})_{6}\right]$, prehnita $\left[\mathrm{Ca}_{2} \mathrm{Al}_{2} \mathrm{Si}_{3} \mathrm{O}_{10}(\mathrm{OH})_{2}\right]$, caolinita $\left[\mathrm{Al}_{4}\left(\mathrm{Si}_{4} \mathrm{O}_{10}\right)(\mathrm{OH})_{8}\right]$, barita $\left(\mathrm{BaSO}_{4}\right)$, sericita $\left[\mathrm{KAl}_{2}\left(\mathrm{AlSi}_{3} \mathrm{O}_{10}\right)(\mathrm{OH})_{2}\right]$, adularia $\left(\mathrm{KAlSi}_{3} \mathrm{O}_{8}\right)$ y epidota $\left[\mathrm{Ca}_{2}(\mathrm{~F}, \mathrm{OH}) \mathrm{Fe}_{2}\left(\mathrm{SiO}_{4}\right)_{3}\right]$ en general son menos abundantes que los minerales antes mencionados (Geyne et al., 1963). La morfología y composición de la mineralización, así como su relación con las rocas encajonantes han permitido definir al yacimiento como epitermal de vetas tipo adularia-sericita bajo en sulfuros (Black, 1963; Geyne et al., 1963; Drier, 1976; Hayba et 
al., 1985; Silberman \& Berger, 1985; Heald et al., 1987; Bonham, 1988). En el distrito existen cuatro presas de jales, localizadas una en la parte sureste (Presa Dos Carlos) y dos al suroeste de la ciudad de Pachuca (Presa Sur I y Presa Sur II) y una cuarta al noreste, aproximadamente a $12 \mathrm{~km}$ de la ciudad de Pachuca (Presa Omitlán) (Fig. 1). La superficie cubierta por los depósitos de jales se ha incrementado hacia la porción sur de la ciudad de Pachuca. En el año de 1959, las presas Sur I y II contenían 55 millones de toneladas con tamaño de partícula del 58\% a -200 mallas y composición química promedio de 47,6 g/ton de Ag, 0,16 g/ ton de $\mathrm{Au}, 0,2 \%$ de $\mathrm{Zn}, 0,1 \%$ de $\mathrm{Pb}, 0,01 \%$ de $\mathrm{Cu}$ y $2,0 \%$ de $\mathrm{Fe}$, de acuerdo a análisis químicos reportados por la Comisión de Fomento Minero (Geyne et al., 1963). Actualmente el volumen aproximado de jales es de 9650 millones de metros cúbicos, distribuidos en una área aproximada de 400 hectáreas (Castañeda, 2002). Las presas de jales están situadas en una capa de suelo, el cual no tuvo un tratamiento previo al depósito. El suelo descansa sobre el paquete volcánico basáltico. Debido a que estas presas se ubican en la planicie, los arroyos y ríos locales son de tipo intermitente, por lo que su interacción con el sistema hidrológico es mínima.

\section{Muestreo}

Se realizó el muestreo en los taludes y en forma de secciones con rumbo NE-SW y NW-SE, (equidistancia de 50 y $100 \mathrm{~m}$, en función de la dimensión de la presa) en las cuatro presas del distrito. Esto permitió conocer el tipo de mineralogía y su comportamiento durante los procesos de alteración causados por la meteorización. Se colectaron un total de 177 muestras: 33 de la presa Omitlán, 55 de Dos Carlos, 10 de Sur I, y 79 de Sur II. El muestreo sobre los taludes fue de tipo canal con una longitud de 1,0 a $1,50 \mathrm{~m}$ por $0,30 \mathrm{~m}$ de espesor y el de las secciones en forma de barrenos con una profundidad de 0,70 $\mathrm{m}$ y un peso aproximado de $1,5 \mathrm{~kg}$ de muestra. Los espesores medidos en las presas son entre 8,0 y $10,0 \mathrm{~m}$ (taludes) y la toma de las muestras fue en la parte basal, central y superior del canal. Los estratos muestreados pertenecen a la deposición antigua y reciente ya que estos residuos han sido removidos de una presa a otra. La referencia del punto de muestreo se realizó mediante un posicionador geográfico satelital (GPS). Las muestras se tomaron con palas de plástico y para evitar la contaminación se almacenaron en bolsas de plástico y se etiquetaron para su posterior identificación. El manejo de las muestras fue cuidadoso por los efectos de contaminación que puedan ocurrir durante su colección, transporte y manipulación para los diferentes análisis. La preparación de las muestras de jales consistió en secado a temperatura ambiente para evitar el proceso de alteración, en un cuarto cerrado y libre de corrientes de aire para evitar la contaminación entre muestras y por partículas de polvo.

\section{Análisis químico}

Los análisis químicos de sólidos (jales) se realizaron en el Servicio Geológico Mexicano (SGM). El análisis químico cuantitativo para determinar concentraciones de elementos traza y mayores calculados en base a óxidos se realizó por espectrometría de plasma de inducción acoplada (ICP), previa digestión de las muestras en un horno de microondas marca CEM, utilizando una mezcla $\left(\mathrm{HCl}+\mathrm{HNO}_{3}\right.$, con una relación de 3:1). Las muestras fueron analizadas en un espectrómetro de plasma Termo Jarrel Ash. El control de calidad de los análisis se aseguró mediante el empleo de estándares 1595-006, 1595004 y 1595-005 High Purity, por cada 10 muestras analizadas y las concentraciones expresadas en $\mathrm{mg} / \mathrm{kg}$. El análisis químico para $\mathrm{SO}_{4}, \mathrm{CO}_{3}$, $\mathrm{S}$ total y elemental fue realizado en los laboratorios del Centro de Investigación de Servicios Industriales Peñoles. Los análisis químicos de los lixiviados recuperados en las pruebas diagnósticas de movilidad por los métodos NOM-053ECOL-1993 y EPA1312 fueron realizados por el Laboratorio Químico del Instituto de Metalurgia de la Universidad Autónoma de San Luis Potosí, por los métodos de espectrometría de absorción 
atómica de flama (AAF) para cuantificar $\mathrm{Cd}, \mathrm{Pb}$, $\mathrm{Zn}, \mathrm{Cu}, \mathrm{Mn}, \mathrm{Fe}, \mathrm{Ni}, \mathrm{Ag}$, las concentraciones están expresadas en $\mathrm{mg} / \mathrm{L}$.

\section{Análisis mineralógico}

La caracterización mineralógica incluye análisis por microscopía óptica de polarización (MOP), microscopía electrónica de barrido (MEB), microsonda electrónica (ME) y difracción de rayos-X (DRX). Se determinaron especies minerales primarias (asociaciones minerales del yacimiento), productos de alteración (asociaciones minerales secundarias no solubles al agua) y fases minerales mayoritarias, minoritarias e indicios. Para los análisis de MOP, MEB y ME se prepararon secciones pulidas embebidas en resina epóxica, pulidas con abrasivos y pasta de diamante para obtener superficies de espejo, para observar texturas, asociaciones mineralógicas, intercrecimientos y halos de alteración (fases secundarias en el MOP). Para los microanálisis al MEB y la ME, después de su pulido se cubrieron con una fina capa de grafito. Los análisis por MOP y MEB se realizaron con los microscopios Olympus modelo BH41 y Leica modelo DC300 y microscopio Phillips modelo XL30, respectivamente. Los análisis por ME se realizaron en un equipo CAMECA modelo SX50. El tiempo (de conteo) para el microanálisis fue de 10 segundos para cada elemento ( $\mathrm{S}, \mathrm{Fe}, \mathrm{As}, \mathrm{Zn}, \mathrm{Cd}, \mathrm{Cu}, \mathrm{Ni}, \mathrm{Pb}$, $\mathrm{Co}, \mathrm{Mn}, \mathrm{Sb}, \mathrm{Ag}$ y Bi), se utilizaron estándares de compuestos químicos y puros con límites de detección de 0,3 hasta 1,6 dependiendo del tiempo de conteo y voltaje $(20 \mathrm{Kv}$ ) en las líneas $\mathrm{K} \alpha, \mathrm{L} \alpha$ y $\mathrm{M} \alpha$ con una corrección de iteraciones ZAF.

\section{Análisis estadístico en componentes princi- pales $(\mathrm{ACP})$}

El análisis estadístico en componentes principales (ACP) se utilizó para el tratamiento y reducción de variables, que en este caso corresponden a los valores de los análisis químicos de las muestras de jales. El propósito es encontrar los componentes estadísticos que expliquen la principal asociación o variación dentro de un grupo de datos. Normalmente, solo los componentes con valores próximos a 1 son interesantes (Danielsson et al., 1999). Estudios con aplicaciones geoquímicas para identificación de fuentes de contaminación por elementos pesados utilizando este método han sido reportados por Aragón et al. (1999), Facchinelli et al. (2001) y Reimann et al. (2002). Se realizó el análisis estadístico con los datos del análisis químico multielemental de las 177 muestras de las cuatro presas del distrito. Primero se realizó en forma individual (por presa) y luego en conjunto (las cuatro presas). Para este análisis se utilizó el software XL Stat, acoplado al MS Excel para Windows.

\section{Diagnóstico de la movilidad de metales}

Se realizaron dos tipos de pruebas en 31 muestras de las presas de jales (por duplicado). El diagnóstico de pruebas de movilidad por el método de la NOM-053-ECOL-1993, representa la movilidad bajo condiciones de confinamiento tipo relleno sanitario o en suelos con alta capacidad de producción de ácidos orgánicos. El extractante empleado en esta prueba fue una solución de ácido acético a pH 2,9 ó 4,9 dependiendo del pH original de la muestra. El segundo método utilizado es el EPA-1312, que representa la movilidad de los elementos potencialmente tóxicos (EPTs) solubles por contacto con agua meteórica tipo lluvia ácida en planicies o llanuras. El extractante que se utilizó fue agua desionizada a un $\mathrm{pH} 4,2$ ó 5,0 ajustado con una mezcla de $\mathrm{HNO}_{3}-\mathrm{H}_{2} \mathrm{SO}_{4}$ (solución que simula el agua meteórica ácida). Los resultados obtenidos de ambas pruebas de diagnóstico de movilidad se explican en función de la disolución de los metales bajo condiciones no oxidantes y se comparan con los límites máximos permisibles (LMP) establecidos por la Norma Oficial Mexicana NOM-052-ECOL-1993. Los resultados de estas pruebas se interpretan en función de la calidad química de los lixiviados producidos y de la movilidad potencial de contaminantes en función del $\mathrm{pH}$ de la muestra. 


\section{RESULTADOS}

\section{Composición química de residuos sólidos (jales)}

En el cuadro 1 se presentan los contenidos de $\mathrm{Pb}, \mathrm{Zn}, \mathrm{Cu}, \mathrm{Fe}, \mathrm{Mn}, \mathrm{Cd}, \mathrm{As}, \mathrm{Bi}, \mathrm{Ag}, \mathrm{Sr}, \mathrm{Ba}$, $\mathrm{Ni}$, Co y $\mathrm{Cr}$, con valores mínimo, máximo, promedio, desviación estándar y límite de detección. Los jales de este tipo de mineralización contienen concentraciones que varían desde centenas hasta millares de $\mathrm{mg} / \mathrm{kg}$. Su concentración es variable de una muestra a otra, pero sobre todo de una presa de jales a otra. De los elementos considerados como potencialmente tóxicos (EPTs) con concentraciones ambientalmente significativas, sobresale el $\mathrm{Pb}$, aunque elementos como $\mathrm{Mn}, \mathrm{Zn}, \mathrm{Cu}$ y $\mathrm{Cd}$, podrían presentar un riesgo si se presentan procesos de concentración. En general, la mayoría de los EPTs se presentan en mayor concentración (a excepción del As) en la presa de jales Omitlán: 1800 a $13000 \mathrm{mg} / \mathrm{kg} \mathrm{Pb}$ (3 551 $1943 \mathrm{mg} / \mathrm{kg}) ; 2$ 500 a $20700 \mathrm{mg} / \mathrm{kg} \mathrm{Mn}(14540 \pm 3752 \mathrm{mg} / \mathrm{kg}) ;$ 1200 a $9000 \mathrm{mg} / \mathrm{kg} \mathrm{Zn} \mathrm{(6} 675 \pm 1484 \mathrm{mg} / \mathrm{kg}) ;$ 51 a $1171 \mathrm{mg} / \mathrm{kg} \mathrm{Cu}(417 \pm 311 \mathrm{mg} / \mathrm{kg}): 2500 \mathrm{a}$ $20700 \mathrm{mg} / \mathrm{kg} \mathrm{Mn}(14500 \pm 3752 \mathrm{mg} / \mathrm{kg})$ y $3,7 \mathrm{a}$ $40,3 \mathrm{mg} / \mathrm{kg} \mathrm{Cd}(28 \pm 7 \mathrm{mg} / \mathrm{kg})$. Por otra parte, se observa que el $\mathrm{Cu}$ presenta concentraciones significativas también en las presas Sur I, de 76 a 304 $\mathrm{mg} / \mathrm{kg}(139 \pm 64 \mathrm{mg} / \mathrm{kg})$ y Sur II, de 4 a $1468 \mathrm{mg} /$ $\mathrm{kg}(185 \pm 187,37 \mathrm{mg} / \mathrm{kg})$. El As es más abundante en la presa Sur I con valores de 11 a $168 \mathrm{mg} / \mathrm{kg}$ $(40 \pm 49,82 \mathrm{mg} / \mathrm{kg})$. La alta desviación estándar

Cuadro 1

Análisis químicos de elementos traza por espectrometría de plasma de inducción acoplada (ICP) de las muestras de jales de las presas Dos Carlos, Sur I, Sur II y Omitlán del distrito minero Pachuca - Real del Monte

\begin{tabular}{|c|c|c|c|c|c|c|c|c|c|c|c|c|c|c|}
\hline $\begin{array}{l}\text { Muestras/ } \\
\mathrm{mg} / \mathrm{kg} \\
\text { Límite } \\
\text { detección }\end{array}$ & $\begin{array}{c}\mathrm{Pb} \\
0,02\end{array}$ & $\begin{array}{c}\mathrm{Zn} \\
0,02\end{array}$ & $\begin{array}{c}\mathrm{Cu} \\
0,01\end{array}$ & $\begin{array}{c}\mathrm{Fe} \\
0,03\end{array}$ & $\begin{array}{c}\mathrm{Mn} \\
0,02\end{array}$ & $\begin{array}{c}\mathrm{Cd} \\
0,005\end{array}$ & $\begin{array}{c}\text { As } \\
0,0004\end{array}$ & $\begin{array}{c}\mathrm{Bi} \\
0,003\end{array}$ & $\begin{array}{c}\mathrm{Ag} \\
0,01\end{array}$ & $\begin{array}{c}\mathrm{Sr} \\
0,01\end{array}$ & $\begin{array}{c}\mathrm{Ba} \\
0,01\end{array}$ & $\begin{array}{c}\mathrm{Ni} \\
0,01\end{array}$ & $\begin{array}{c}\text { Co } \\
0,01\end{array}$ & $\begin{array}{c}\mathrm{Cr} \\
0,01\end{array}$ \\
\hline \multicolumn{15}{|c|}{ Dos Carlos } \\
\hline Mínimo & 160 & 230 & 30 & 17900 & 700 & 0,7 & 0,0 & 0,0 & 15,0 & 62,0 & 252,4 & 6,2 & 0,6 & 93,0 \\
\hline Máximo & 1300 & 2400 & 248 & 48038 & 7700 & 19,5 & 30,0 & 8,3 & 110,0 & 145,2 & 879,0 & 80,0 & 13,0 & 22,0 \\
\hline Promedio & 340 & 688,6 & 66,3 & 31020 & 3925 & 4,1 & 4,8 & 1,3 & 43,3 & 111,1 & 345,0 & 21,3 & 5,7 & 34,5 \\
\hline Desv.Están. & 188,9 & 383,1 & 38,7 & 6221,3 & 1939,4 & 3,7 & 7,7 & 2,3 & 19,9 & 19,5 & 129,5 & 15,0 & 2,7 & 14,5 \\
\hline \multicolumn{15}{|c|}{ Presa Sur I } \\
\hline Mínimo & 700 & 1500 & 76 & 15800 & 2400 & 6,1 & 11,0 & 0,0 & 31,0 & 131,0 & 443,0 & 43,0 & 7,0 & 41,0 \\
\hline Máximo & 6600 & 3100 & 304 & 56800 & 9100 & 20,6 & 168,0 & 1,5 & 190,6 & 207,0 & 752,0 & 75,0 & 10,0 & 164,0 \\
\hline Promedio & 1910 & 2540 & 139 & 26090 & 5600 & 13,0 & 40,2 & 0,4 & 89,5 & 161,2 & 509,2 & 60,9 & 8,4 & 65,7 \\
\hline Desv.Están. & 1701,3 & 424,8 & 63,9 & 128889 & 146572 & 4,2 & 49,8 & 0,5 & 58,1 & 28,6 & 103,4 & 9,5 & 1,2 & 38,3 \\
\hline \multicolumn{15}{|c|}{ Presa II } \\
\hline Mínimo & 2350 & 196 & 4 & 14900 & 4100 & 0,5 & 7,0 & 0,4 & 20,0 & 75,8 & 196,5 & 18,6 & 1,6 & 27,7 \\
\hline Máximo & 3460 & 6853 & 1468 & 58657 & 10100 & 88,2 & 49,4 & 11,4 & 376,9 & 204,2 & 573,0 & 73,0 & 14,0 & 120,0 \\
\hline Promedio & 1205,5 & 2914,1 & 184,9 & 26348,1 & 6651,9 & 14,2 & 12,0 & 1,5 & 44,5 & 119,1 & 294,7 & 18,8 & 6,5 & 42,3 \\
\hline Desv.Están. & 1113,0 & 2659,2 & 187,4 & 91599,6 & 17400,9 & 12,5 & 11,1 & 2,5 & 44,7 & 28,2 & 95,1 & 11,9 & 2,9 & 17,4 \\
\hline \multicolumn{15}{|c|}{ Omitlán } \\
\hline Mínimo & 3770 & 1200 & 51 & 11000 & 2500 & 3,7 & 0,0 & 0,0 & 42,1 & 82,3 & 155,3 & 6,7 & 1,3 & 13,4 \\
\hline Máximo & 5450 & 9006 & 1171 & 48600 & 18600 & 40,3 & 24,5 & 2,6 & 201,0 & 164,0 & 388,0 & 38,0 & 6,0 & 67,0 \\
\hline Promedio & 3551,2 & 6674,6 & 416,9 & 26450 & 145424 & 27,7 & 8,4 & 0,6 & 77,0 & 114,0 & 240,8 & 13,5 & 3,5 & 20,7 \\
\hline Desv.Están. & 1942,7 & 1484,5 & 311,0 & 9236,8 & 3751,6 & 6,8 & 9,8 & 1,0 & 35,2 & 21,7 & 78,3 & 9,7 & 1,2 & 11,2 \\
\hline
\end{tabular}


del As en Sur I debe obedecer a la menor densidad de muestreo (10 muestras). La concentración de As es también significativa en la presa Sur II, con valores que van desde 1 hasta $49 \mathrm{mg} / \mathrm{kg}$, con un promedio de $12 \mathrm{mg} / \mathrm{kg}$ y una desviación estándar de $\pm 11 \mathrm{mg} / \mathrm{kg}$, presentando niveles más heterogéneos en concentración. Otros elementos como el $\mathrm{Sr}, \mathrm{Ba}, \mathrm{Ni}, \mathrm{Co}$ y $\mathrm{Cr}$ presentan concentraciones relativamente homogéneas en las cuatro presas de jales del distrito, siendo ligeramente mayor la concentración de Ba en la presa Sur I con valores de 395 a $752 \mathrm{mg} / \mathrm{kg}(509 \pm 103,40 \mathrm{mg} / \mathrm{kg})$ y de $\mathrm{Cr}$ en la presa Sur II con valores que van desde 17,35 a $120,0 \mathrm{mg} / \mathrm{kg}(42 \pm 8 \mathrm{mg} / \mathrm{kg})$. Las concentraciones de $\mathrm{Bi}$ y Ag son muy heterogéneas en todas las presas del distrito, se observa un promedio y desviación estándar de $1,0 \pm 2,0 \mathrm{mg} / \mathrm{kg}$ de $\mathrm{Bi}$ en las cuatro presas. La plata presenta mayores concentraciones en la presa Omitlán con valores de 42 a $201 \mathrm{mg} / \mathrm{kg}(77 \pm 35 \mathrm{mg} / \mathrm{kg})$.

Con respecto a los elementos mayores calculados en base a óxidos (Cuadro 2), se puede observar que el $\mathrm{Al}_{2} \mathrm{O}_{3}, \mathrm{~K}_{2} \mathrm{O}$ y $\mathrm{SiO}_{2}$ son más o menos homogéneos en las cuatro presas con valores promedio y desviación estándar de 8,12 y $\pm 2,38 \%$ de $\mathrm{Al}_{2} \mathrm{O}_{3} ; 2,93$ y $\pm 0,691 \%$ de $\mathrm{K}_{2} \mathrm{O}$ y 73,11 y \pm $9,283 \%$ de $\mathrm{SiO}_{2}$. Lo cual representaría la naturaleza del yacimiento (mena y roca encajonante), vetas de matriz cuarzosa encajonadas en rocas de composición ácida a intermedia (aluminosilicatos) y alteraciones de tipo hidrotermal derivadas de feldespato potásico "sanidina y adularia" (caolín, montmorrillonita). El $\mathrm{FeO}$ y el $\mathrm{CO}_{3}$ presentan valores heterogéneos con un promedio y

Cuadro 2

Análisis químicos de elementos mayores calculados en base a óxidos por espectrometría de plasma de inducción acoplada (ICP) de las muestras de jales de las presas Dos Carlos, Sur I, Sur II y Omitlán del distrito minero Pachuca - Real del Monte

\begin{tabular}{|c|c|c|c|c|c|c|c|c|c|c|c|}
\hline $\begin{array}{c}\text { Muestra/\% } \\
\text { Límite detec- } \\
\text { ción }\end{array}$ & $\begin{array}{c}\mathrm{Al}_{2} \mathrm{O}_{3} \\
0,02\end{array}$ & $\begin{array}{l}\mathrm{CaO} \\
0,01\end{array}$ & $\begin{array}{l}\mathrm{K}_{2} \mathrm{O} \\
0,05\end{array}$ & $\begin{array}{c}\mathrm{MgO} \\
0,02\end{array}$ & $\begin{array}{c}\mathrm{MnO} \\
0,04\end{array}$ & $\begin{array}{l}\mathrm{FeO} \\
0,03\end{array}$ & $\begin{array}{c}\mathrm{Na}_{2} \mathrm{O} \\
0,08\end{array}$ & $\begin{array}{l}\mathrm{TiO}_{2} \\
0,01\end{array}$ & $\begin{array}{l}\mathrm{SiO}_{2} \\
0,01\end{array}$ & $\begin{array}{c}\mathrm{CO}_{3} \\
-\end{array}$ & $\begin{array}{c}\mathrm{SO}_{4} \\
-\end{array}$ \\
\hline \multicolumn{12}{|c|}{ Presa Dos Carlos } \\
\hline Mínimo & 4,82 & 0,41 & 1,76 & 0,22 & 0,09 & 2,30 & 0,10 & 0,14 & 48,67 & 0,22 & 0,31 \\
\hline Máximo & 14,29 & 3,20 & 3,69 & 0,98 & 0,99 & 6,18 & 0,54 & 0,48 & 82,40 & 4,50 & 4,60 \\
\hline Promedio & 10,49 & 1,55 & 2,78 & 0,54 & 0,51 & 3,99 & 0,19 & 0,28 & 75,83 & 1,05 & 1,37 \\
\hline Desv.Están. & 2,23 & 0,70 & 0,53 & 0,18 & 0,25 & 0,80 & 0,10 & 0,08 & 6,24 & 5,09 & 0,98 \\
\hline \multicolumn{12}{|c|}{ Presa Sur I } \\
\hline Mínimo & 5,65 & 2,74 & 2,87 & 0,66 & 0,31 & 0,31 & 0,69 & 0,23 & 50,50 & 2,10 & 2,90 \\
\hline Máximo & 10,85 & 5,20 & 3,90 & 1,65 & 1,18 & 1,18 & 1,33 & 0,40 & 55,71 & 12,50 & 13,30 \\
\hline Promedio & 7,83 & 3,39 & 3,48 & 1,06 & 0,72 & 0,72 & 0,96 & 0,32 & 55,82 & 3,84 & 4,79 \\
\hline Desv.Están. & 1,53 & 0,93 & 0,38 & 0,39 & 0,25 & 0,25 & 0,24 & 0,06 & 3,64 & 3,36 & 3,56 \\
\hline \multicolumn{12}{|c|}{ Presa II } \\
\hline Mínimo & 4,16 & 1,49 & 1,68 & 0,50 & 1,37 & 1,53 & 0,33 & 0,18 & 52,07 & 1,20 & 0,04 \\
\hline Máximo & 9,95 & 4,53 & 4,45 & 1,59 & 0,50 & 7,55 & 1,15 & 0,43 & 80,30 & 4,33 & 6,20 \\
\hline Promedio & 7,53 & 3,29 & 3,14 & 0,88 & 0,85 & 3,39 & 0,58 & 0,30 & 72,38 & 3,60 & 1,32 \\
\hline Desv.Están. & 1,25 & 0,57 & 0,82 & 0,27 & 0,22 & 1,18 & 0,20 & 0,05 & 9,75 & 3,97 & 2,75 \\
\hline \multicolumn{12}{|c|}{ Omitlán } \\
\hline Mínimo & 3,28 & 1,54 & 1,98 & 0,90 & 0,32 & 1,53 & 0,28 & 0,17 & 58,74 & 1,19 & 0,07 \\
\hline Máximo & 7,81 & 6,67 & 3,16 & 1,50 & 2,54 & 6,25 & 1,07 & 0,23 & 83,50 & 4,59 & 4,40 \\
\hline Promedio & 5,68 & 3,90 & 2,54 & 1,20 & 1,47 & 2,08 & 0,56 & 0,21 & 75,56 & 2,84 & 0,82 \\
\hline Desv.Están. & 1,32 & 1,33 & 0,27 & 0,26 & 0,37 & 0,86 & 0,24 & 0,03 & 7,72 & 1,16 & 1,25 \\
\hline
\end{tabular}


desviación estándar de 3,57 y $\pm 1,320 \%$ y 2,68 y $\pm 3,706 \%$ respectivamente, que puede explicarse en el primer caso por algunos niveles muestreados más oxidados. Sobre todo en las muestras de la presa Dos Carlos, donde se observa una fuerte alteración (precipitación) del residuo (jal) a óxidos e hidróxidos de hierro, ya que estos presentan una coloración que va de color crema a amarillo rojizo y en ocasiones a color café oscuro. Por otra parte, el hierro también representaría tanto minerales de la mena (pirita, calcopirita, bornita) como a minerales de la roca encajonante (piroxenos y anfíboles). Para el segundo caso, la presencia de carbonatos en las vetas (bustamita y calcita) podría explicarse como un proceso hidrotermal tardío de mineralización. El MnO también presenta valores muy homogéneos en las cuatro presas, sin embargo, se observa una mayor concentración de manganeso en la presa Omitlán, con valores que van de $1,11 \%$ a $2,45 \%$, con un promedio y desviación estándar de 1,47 y $\pm 0,423 \%$. Esto nos representaría minerales de las estructuras mineralizadas como la rodonita y la bustamita. $\mathrm{El} \mathrm{SO}_{4}$ presenta valores heterogéneos con un promedio y desviación estándar de 1,44 y \pm 2,294\%, siendo mayor la concentración en la presa Sur I y correspondería principalmente a la formación de sulfatos, minerales generalmente hidratados. La concentración de sulfatos corresponde por una parte a la oxidación férrica de los minerales sulfurosos primarios como la pirita y pirrotita y por otra puede ser también debido a los procesos de intemperismo en la presa de jales.

\section{Caracterización mineralógica de jales}

\section{Difracción de rayos $X$}

El análisis por difracción de rayos $\mathrm{x}$ (DRX) permitió determinar en las cuatro presas del distrito como fase mineral mayoritaria al cuarzo $\left(\mathrm{SiO}_{2}\right)$, lo cual depende de la naturaleza metalogenética del yacimiento. También se determinaron algunas fases minerales minoritarias como yeso $\left(\mathrm{CaSO}_{4} 2 \mathrm{H}_{2} \mathrm{O}\right)$, calcita $\left(\mathrm{CaCO}_{3}\right)$, escasa alunita $\left.\left[\mathrm{K}_{2} \mathrm{Al}_{6}(\mathrm{OH})_{12}\left(\mathrm{SO}_{4}\right)_{4}\right)\right]$ y pirita $\left(\mathrm{FeS}_{2}\right)$. En la presa Dos Carlos la mayoría de las muestras presentan una fase mineral mayoritaria de cuarzo, que representaría la matriz de la mineralización (vetas cuarzosas) y solo se observan indicios de los minerales que constituyen la roca encajonante. Las presas Sur I, Sur II y Omitlán presentan la misma fase mineral mayoritaria pero con relictos de la mineralización metálica (pirita, esfalerita y galena), además de la presencia de minerales de la roca encajonante (albita y sanidina) y minerales característicos de alteración (alunita, yeso y jarosita).

\section{Microscopía óptica de polarización (MOP)}

Las muestras observadas al MOP presentan abundantes fragmentos de cristales de cuarzo, silicatos y escasa calcita emplazados en una matriz silícea. La mineralización metálica es muy escasa, aproximadamente del 1 al 5\% en abundancia de mineralización con respecto a la superficie de la muestra. Está constituida principalmente por pirita $\left(\mathrm{FeS}_{2}\right)$ que es la más abundante, goethita $(\mathrm{FeO}(\mathrm{OH}))$-hematita $\left(\mathrm{Fe}_{2} \mathrm{O}_{3}\right)($ ?), escasa esfalerita $(\mathrm{ZnS})$ y galena $(\mathrm{PbS})$ y más escasa aún calcopirita $\left(\mathrm{CuFeS}_{2}\right)$ y titanita $\left(\mathrm{CaTiSiO}_{5}\right)$. La mineralogía metálica está asociada a cuarzo $\left(\mathrm{SiO}_{2}\right)$ o calcita $\left(\mathrm{CaCO}_{3}\right)$ y silicatos.

La pirita presenta una morfología que va de subhedral a euhedral, principalmente en partículas liberadas sobre todo en la fracción más fina. En ocasiones se observa con fracturas y bordes de alteración a óxidos y/o hidróxidos de hierro. Presenta inclusiones de galena, esfalerita y calcopirita. También se observa en pequeñas inclusiones dentro de cuarzo, silicatos y en ocasiones asociada a goethita-hematita. La goethita-hematita se encuentra asociada sobre todo a la pirita o en inclusiones dentro de cuarzo, silicato o carbonato y huellas de boxworks. También se observó liberada, lo que puede corresponder a la alteración total de la pirita ya que presenta seudomorfismo. Debe señalarse que esta especie mineral se manejo como un grupo de óxi-hidróxidos de hierro, ya que presentan características muy similares y normalmente son cristales muy pequeños, y es difícil de diferenciar al microscopio óptico de polarización. La esfalerita es muy escasa. En ocasiones se asocia a la pirita y a la calcopirita. 
También se observa libre con inclusiones de pirita o completamente dañada, con fracturas y bordes caóticos probablemente por los reactivos utilizados en el proceso de beneficio. En ocasiones se puede observar que la esfalerita, pirita y calcopirita están incluidas o asociadas a silicatos, en los bordes y fracturas de estos. La galena también es muy escasa y se observa en inclusiones muy finas dentro de la pirita. La calcopirita es mucho más escasa que la galena y la esfalerita, se observa en pequeños cristales liberados con fracturamiento y bordes de alteración a posibles sulfatos de cobre y hierro. En ocasiones se asocia a la pirita, galena y esfalerita en los bordes de los silicatos. Se presenta incluida en hematita-goethita, presentando fracturas rellenas de óxidos de hierro. La titanita se observó también muy escasa liberada ó bien en pequeñas inclusiones dentro de la pirita o cuarzo.

Respecto a los minerales transparentes se observa principalmente abundante cuarzo y silicatos. Estos contienen mineralización metálica (pirita, esfalerita, calcopirita) en forma de inclusiones, agregados y en sus bordes. También se observa calcita y silicatos, como: bustamita, adularia, albita, alunita y sanidina (estos dos últimos determinados por difracción de rayos-X). En ocasiones se observan pequeños cristales de apatito $\left[\mathrm{Ca}_{5}(\mathrm{~F}, \mathrm{Cl}, \mathrm{OH})\left(\mathrm{PO}_{4}\right)_{3}\right]$ como inclusiones dentro de silicatos (rodonita).

\section{Microscopía electrónica de barrido (MEB)}

El análisis por microscopía electrónica de barrido se realizó principalmente en las partículas de pirita. Este mineral es el más abundante y presenta una mayor alteración en fracturas y bordes. La finalidad es determinar la mineralogía secundaria. Sin embargo, se tuvo problemas con los cálculos estequiométricos para determinar especies minerales, ya que los análisis se realizaron en las zonas mencionadas.

En el cuadro 3 se presentan los resultados obtenidos del microanálisis puntual en cristales de pirita y en las fases de alteración determinadas en bordes y fracturas. Los microanálisis puntuales en algunos cristales de pirita $(1,4,7)$ presentan composiciones estequiométricas calculadas en base a tres átomos que corresponden a la fórmula teórica de la misma. Se tuvo una incertidumbre de error de entre 0,02-0,01, la cual no es significativa: $\mathrm{Fe}_{(0,99)} \mathrm{S}_{(2,01)}, \mathrm{Fe}_{(1)} \mathrm{S}_{(2)}=\mathrm{FeS}_{2}$. Los microanálisis en las fases de alteración determinadas en algunos de los bordes y fracturas presentan una composición mineral que corresponde a la asociación mineral goethita-hematita (2, 5, 6 en cuadro 3$)$. El cálculo fue en base a cinco átomos, los cuales corresponden a la fórmula teórica de esta última $\mathrm{Fe}_{2} \mathrm{O}_{3}$ (Fig. 2A). Presentan también una incertidumbre de error mínima de $\left.0,08, \mathrm{Fe}_{(2,08}\right) \mathrm{O}_{(2,92)}$, $\left.\mathrm{Fe}_{(2}\right) \mathrm{O}_{(3)}=\mathrm{Fe}_{2} \mathrm{O}_{3}$. En este caso, se puede tratar de una alteración (oxidación) progresiva de la pirita a goethita-hematita. Otras fases de alteración (3, 8 en cuadro 3 ) presentan una composición heterogénea y compleja por lo que es difícil calcular la estequiometria para asignar a estas composiciones una especie mineral determinada (Fig. 2B). Sin embargo, por los elementos presentes y su contenido en peso porcentual, estos pueden corresponder a una pirita que está siendo alterada a un conjunto de sulfatos de hierro. Entre estos, la que destaca es la melanterita $\left(\mathrm{FeSO}_{4} 7 \mathrm{H}_{2} 0\right)$ (Jambor, 1994). Esta especie resulta normalmente de una alteración intermedia de la pirita o marcasita, se produce fácilmente cuando hay un cierto grado de humedad, precipitación meteórica y está expuesta al aire. La presencia de otros elementos ( $\mathrm{S}, \mathrm{Si}$, $\mathrm{Mg}$ ) que están en pequeñas concentraciones es típico de la precipitación de óxidos y oxihidróxidos de $\mathrm{Fe}$ en mineralogías secundarias. Se presentan otros minerales dentro de la pirita, como el rutilo $\mathrm{TiO}_{2}$, ilmenita $\mathrm{FeTiO}_{3}$ y titanita, $\left[\mathrm{CaTiO}\left(\mathrm{SiO}_{4}\right)\right]$. La titanita ( 9 en cuadro 3 ) se observa como inclusión en pirita fracturada (Fig. 2C), la ilmenita (10 en cuadro 3 ) a lo largo de fracturas, planos y/o clivaje de la pirita (Fig. 2D) y el rutilo (11 en cuadro 3) en inclusiones dentro de la pirita (Fig. 2E). Aunque estas exsoluciones están expuestas en zonas de alteración no se detectó alguna asociación con otros elementos neoformadores de minerales secundarios. Por otra parte, y debido a su biocompatibilidad no se han descubierto casos de toxicidad tanto en el titanio elemental como en el dióxido de titanio. El cuadro 3 presenta los resultados de los microanálisis realizados en cristales de calcopirita. Se obtuvieron composiciones estequiométricas calculadas en base a cuatro átomos 
Cuadro 3

Análisis químico puntual semicuantitativo por microscopia electrónica de barrido (MEB) en cristales de pirita y de calcopirita

\begin{tabular}{|c|c|c|c|c|c|c|c|c|c|c|c|}
\hline \multicolumn{12}{|c|}{ W \% del microanálisis en partículas de pirita } \\
\hline Elemento / punto & 1 & 2 & 3 & 4 & 5 & 6 & 7 & 8 & 9 & 10 & 11 \\
\hline $\mathrm{S}$ & 54,13 & & 37,91 & 54,02 & & 2,67 & 53,96 & 22,93 & & & \\
\hline $\mathrm{Fe}$ & 45,87 & 71,47 & 47,83 & 45,98 & 69,11 & 63,99 & 46,04 & 44,02 & & 29,10 & \\
\hline $\mathrm{O}$ & & 28,53 & 14,26 & & 28,79 & 30,45 & & 28,27 & 34,30 & 26,55 & 38,73 \\
\hline $\mathrm{Si}$ & & & & & 2,10 & 2,08 & & 3,71 & 17,58 & & 1,86 \\
\hline $\mathrm{Mg}$ & & & & & & 0,82 & & & & & \\
\hline $\mathrm{Ti}$ & & & & & & & & & 26,38 & 36,59 & 57,26 \\
\hline $\mathrm{Ca}$ & & & & & & & & 1,07 & 21,74 & 0,51 & 2,15 \\
\hline $\mathrm{Na}$ & & & & & & & & & & & \\
\hline $\mathrm{Al}$ & & & & & & & & & & & \\
\hline $\mathrm{Mn}$ & & & & & & & & & & 7,24 & \\
\hline \multicolumn{12}{|c|}{ W \% del microanálisis en partículas de calcopirita } \\
\hline Elemento / punto & 1 & 2 & 3 & 4 & 5 & 6 & 7 & 8 & 9 & 10 & 11 \\
\hline $\mathrm{S}$ & 35,79 & 17,89 & & 8,2 & 33,62 & 17,89 & 13,21 & 35,74 & 33,62 & & \\
\hline $\mathrm{Fe}$ & 29,51 & 39,34 & 12,85 & 31,32 & 28,50 & 39,34 & 38,57 & 29,38 & 28,50 & & \\
\hline $\mathrm{Cu}$ & 34,71 & 23,48 & & & 32,78 & 23,48 & 12,95 & 34,88 & 32,78 & & \\
\hline $\mathrm{O}$ & & 15,93 & 33,18 & 31,29 & & 15,93 & 27,26 & & & & \\
\hline $\mathrm{Si}$ & & 3,36 & 22,91 & 12,96 & & 3,36 & 3,93 & & & & \\
\hline $\mathrm{Mg}$ & & & 3,26 & & & & & & & & \\
\hline $\mathrm{Al}$ & & & 6,32 & 2,19 & & & & & & & \\
\hline $\mathrm{Ag}$ & & & 9,61 & 8,41 & & & & & & & \\
\hline $\mathrm{Mn}$ & & & 4,35 & & & & & & & & \\
\hline $\mathrm{Ca}$ & & & 1,85 & & & & 4,07 & & & & \\
\hline $\mathrm{Na}$ & & & & 5,64 & & & & & & & \\
\hline
\end{tabular}

que corresponden a la fórmula teórica de la calcopirita $\mathrm{S}_{2} \mathrm{FeCu}(1,5,8,9$ en cuadro 3). Presentan valores de incertidumbre de error que varían en rangos de tolerancia mínimos $0,03-0,01 ; \mathrm{S}_{(2,03)}$, $\mathrm{Fe}_{(0,97)}, \mathrm{Cu}_{(0,99)}, \mathrm{S}_{(2)}, \mathrm{Fe}_{(1)}, \mathrm{Cu}(1)$. Otros microanálisis realizados presentan a la calcopirita fracturada y alterada con diferentes grados de intensidad (2, $3,4,6,7$ en cuadro 3). Sin embargo, los puntos analizados no permitieron establecer alguna especie mineral secundaria específica (Fig. 2F). Podría tratarse de la neoformación de compuestos de tipo sulfato $\mathrm{Cu}-\mathrm{Fe}$ (?).

Los microanálisis realizados en cristales de la esfalerita mostraron resultados estequiométricos similares en todos los puntos analizados y sólo en un análisis se constató la presencia de $\mathrm{Cd}, \mathrm{Fe}$ y Ag presentes en su composición. La esfalerita se presenta fracturada, sin embargo no se observa alteración en sus fracturas y/o bordes.

\section{Microsonda electrónica (ME)}

El objetivo de este análisis es determinar la variedad mineral del óxido y/o hidróxido de hierro formado en los bordes y fracturas (alteración) de la pirita, ya que es el mineral metálico más abundante en el distrito. Así como la concentración de elementos potencialmente tóxicos (EPTs) como el $\mathrm{Co}, \mathrm{Ni}, \mathrm{Cr}, \mathrm{Cd}$, entre otros. 


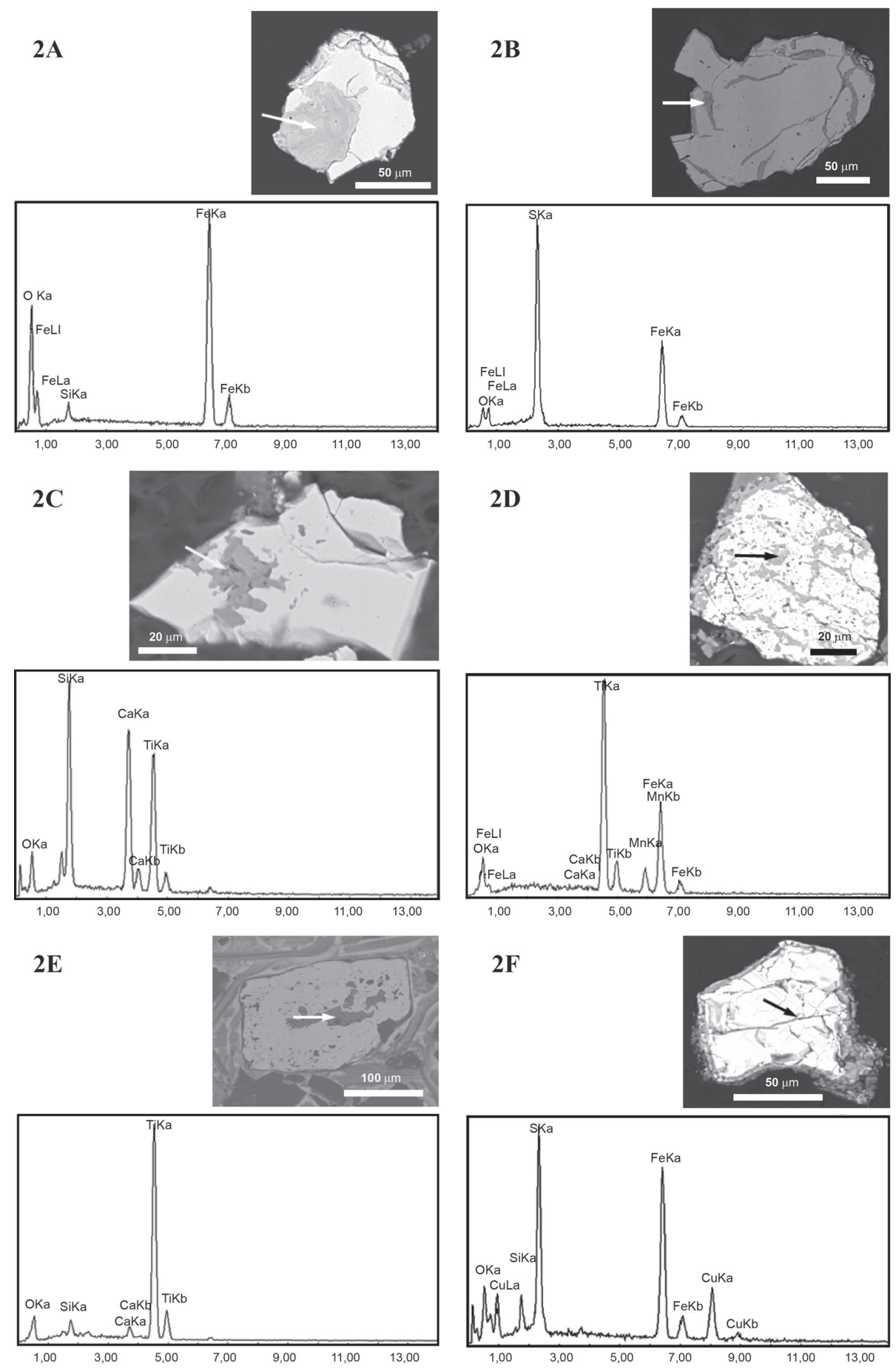

Fig. 2: Fotomicrografía al microscopio electrónico de barrido con electrones retrodispersados. A: alteración progresiva de la pirita a óxidos e hidróxidos de hierro (goethita-hematita), B: pirita con fracturas y bordes rellenos de sulfato de hierro, C: pirita fracturada con inclusiones de titanita, D: pirita fracturada con inclusiones de ilmenita, E: pirita fracturada con inclusiones de rutilo, F: calcopirita fracturada y con bordes rellenos de óxidos de hierro. 
La composición cristaloquímica de la pirita es muy heterogénea, sólo se pudieron comprobar pocos puntos analizados con una composición primaria estequiométrica del mineral. Sin embargo, se confirmó la evidencia de una alteración progresiva, pero sin poder determinar una fase mineral específica, ya que por esta técnica no es posible determinar el hidrógeno y el oxígeno contenido en las muestras analizadas. Se realizaron cálculos estequiométricos con los datos obtenidos en el microanálisis porcentual, normalizando y calculando por diferencia el agua para poder establecer una especie mineral aceptable. Los cálculos son para determinar la asociación limonita-goethita que es la representativa de la alteración (bordes y fracturas). Se puede observar que la mayoría de los análisis presentan deficiencias en cuanto a su estequiometría. Por lo tanto muy pocos puntos de los análisis al realizar los cálculos permiten determinar especies minerales como la goethita con rangos de la suma en peso porcentual calculado en óxidos que van desde $96,77 \%$ hasta $100,64 \%$. Sin embargo, esto se observó sólo en una muestra, en la cual también se pudo determinar una fase mineral de ferrihidrita con valores de la suma en peso porcentual calculado en óxidos que presentan valores entre $92,82 \%$ y $99,56 \%$. También se puede observar en los puntos analizados y calculados en óxidos valores significativos en $\mathrm{Fe}_{2} \mathrm{O}_{3}$ y $\mathrm{SO}_{3}$, lo cual indicaría la formación de sulfato de hierro hidratado del grupo de la melanterita (melanterita $\mathrm{FeSO}_{4} \cdot 7 \mathrm{H}_{2} \mathrm{O}$ y mallardita $\mathrm{MnSO}_{4} \cdot 7 \mathrm{H}_{2} \mathrm{O}$ ).

Respecto a $\mathrm{Ni}, \mathrm{Cr}$ y $\mathrm{Co}$, es el níquel el que se presenta en mayor concentración con valores en peso porcentual desde $0,02 \%$ hasta $4,80 \%$, lo que indica su presencia dentro de la pirita. Por lo tanto, es posible su disolución y/o movilidad causada por los procesos de intemperismo y como consecuencia su incorporación al drenaje del sitio minero. Los otros elementos están presentes pero en concentraciones más bajas. Con respecto al As, es escaso y sólo se detectó en siete puntos analizados con valores desde $0,02 \%$ hasta $1,84 \%$ dentro de cristales de pirita. En el caso de la esfalerita se buscó principalmente la presencia del $\mathrm{Cd}$, que es otro de los elementos potencialmente tóxicos, aunque la esfalerita se encuentra de manera escasa en este distrito. Se observa que la presencia del cadmio y el hierro son constantes, aunque el hierro es el que se presenta en mayor concentración. El Fe presenta valores en concentración desde 0,55 hasta 1,22 en $\%$ de peso. Por el contrario el cadmio es más escaso con concentraciones de 0,30 a 0,47 en $\%$ de peso. También se observan concentraciones significativas de manganeso y cobre, en donde el manganeso puede substituir al zinc y el cobre es el reflejo de la calcopirita.

\section{Análisis estadístico por componentes principales (ACP)}

El análisis estadístico de las concentraciones de elementos traza se realizó para cada presa de jales, así como para el conjunto de las cuatro presas. La finalidad es hacer comparaciones en cuanto a la correlación entre las asociaciones de elementos. El análisis estadístico incluye el cálculo de la correlación lineal y el análisis por ACP.

La matriz de correlación entre pares de elementos reporta una alta correlación entre $\mathrm{Zn}-\mathrm{Cd}$ $(0,90)$ y $\mathrm{Pb}-\mathrm{Cu}(0,90)$ para la presa Omitlán. En la presa Dos Carlos se observa una alta correlación entre Zn-Cd $(0,91)$. Para la presa Sur I se tiene una alta correlación entre $\mathrm{Fe}-\mathrm{Cr}(0,96)$; $\mathrm{Pb}-\mathrm{Cr}(0,93)$ y $\mathrm{Fe}-\mathrm{Pb}(0,91)$. En la presa Sur II existe alta correlación entre $\mathrm{Zn}-\mathrm{Cd}(0,99)$; $\mathrm{Cu}$ $\mathrm{Cd}(0,95) ; \mathrm{Zn}-\mathrm{Cu}(0,94)$ y $\mathrm{Fe}-\mathrm{Sr}(0,93)$. En el conjunto se obtuvieron los siguientes resultados: se mantienen la alta correlación entre $\mathrm{Zn}$ $\mathrm{Cd}(0,98)$; una buena correlación entre $\mathrm{Zn}-\mathrm{Cu}$ $(0,81)$ y $\mathrm{Cu}-\mathrm{Cd}(0,81)$; una relativa correlación entre $\mathrm{Ba}-\mathrm{Ni}(0,70)$ y otras de menor magnitud entre Mn-Zn $(0,68), \mathrm{Co}-\mathrm{Cr}(0,67), \mathrm{Pb}-\mathrm{Zn}(0,63)$, $\mathrm{Mn}-\mathrm{Cd}(0,60), \mathrm{Pb}-\mathrm{Cd}(0,60)$. De acuerdo a estos resultados se observa que la correlación entre $\mathrm{Zn}$ y Cd es altamente significativa dentro de las presas. Esto se puede explicar por la típica presencia de $\mathrm{Cd}$ en la estructura de la esfalerita $(\mathrm{Zn}, \mathrm{Cd}) \mathrm{S}$ ó bien como exsolución a manera de inclusión de greenockita (CdS) dentro de la esfalerita. Se observan otras correlaciones entre pares de elementos como: $\mathrm{Zn}-\mathrm{Cu}, \mathrm{Cu}-\mathrm{Cd}, \mathrm{Fe}-\mathrm{Cu}$ y $\mathrm{Pb}-\mathrm{Cu}$ que puede tratarse de la asociación esfalerita-calcopirita-galena, representando parte de la mineralogía primaria del yacimiento. Otras 
correlaciones relativas y de menor magnitud (Ba-Ni, Mn-Zn, Co-Cr, Ni-Co, Pb-Zn, Fe-Co) se explicarían como presencia de barita, asociación de braunita-bustamita-manganita-rodonita con esfalerita y galena y presencia como impurezas ó soluciones sólidas de Cr, Co y Ni dentro de la magnetita y de la pirita, característica que puede ser común de estas mineralogías.

En el análisis factorial por componentes principales (ACP) para cada presa, se obtuvo un alto porcentaje de varianza acumulada para los tres primeros factores $(>70 \%)$. Se construyeron gráficas tipo nube de puntos para observar la asociación entre variables de cada presa. En las presas Omitlán, Dos Carlos y Sur II, las asociaciones geoquímicas entre la mayoría de las variables está estadísticamente bien explicada, solo en la presa Sur I no se observa una asociación mineralógica aceptable. En el conjunto se obtuvo un porcentaje de varianza acumulada del $65 \%$ para los tres primeros factores, lo cual podría explicarse por la gran variación en la composición química entre las distintas presas de jales del distrito. En el círculo de correlación del conjunto se observan las siguientes asociaciones geoquímicas: $\mathrm{Ba}-\mathrm{Cr}-$ $\mathrm{Ni}-\mathrm{Co}$ y $\mathrm{Cu}-\mathrm{Cd}-\mathrm{Zn}$; de las cuales, la asociación $\mathrm{Cu}-\mathrm{Cd}-\mathrm{Zn}$ está estadísticamente mejor explicada. Otras asociaciones como As-Sr y Fe-Ag serían menos significativas en estos factores. La asociación $\mathrm{Zn}-\mathrm{Cd}-\mathrm{Cu}$ se trata de la asociación geoquímica cadmio-esfalerita y la asociación mineralógica esfalerita-calcopirita. En cambio, la asociación Ba-Cr-Ni-Co, podría corresponder a impurezas o soluciones sólidas dentro de la magnetita y la pirita y a la barita que se encuentra como mineral de ganga. Probablemente estos elementos y el hierro reflejan el comportamiento geoquímico calcófilo y compatible de estos elementos. $\mathrm{La}$ pirita puede contener pequeñas cantidades de $\mathrm{Ni}$ y Co. Algunos análisis han mostrado considerable Ni y una serie completa de soluciones sólidas existe entre la pirita y la bravoita (Klein, 2002). Según Jambor (1994), hay numerosos elementos traza, tales como $\mathrm{Ni}$, Co y $\mathrm{Cr}$ que se encuentran entre poros intersticiales, por lo que podría corresponder a sales solubles. Esto es, sulfatos de níquel formados durante la disolución de los minerales primarios y precipitados como minerales secundarios en los poros. La solución sólida del $\mathrm{Ni}$, sustituyendo otro elemento, se espera que suceda, pero no ha sido aún bien documentada (Alpers et al., 1994).

\section{Movilidad química de metales}

Las pruebas de diagnóstico de la movilidad de metales (Cd, Pb, Zn, Cu, Mn, Fe, Ni, Ag, Co y As) por los métodos NOM-053-ECOL/1993 y EPA1312, se realizaron en 31 muestras ( 8 de Omitlán, 8 de Dos Carlos, 4 de Sur I, y 11 de Sur II). Los lixiviados o extractos recuperados para cada muestra en ambas tipos de pruebas se compararon con los límites máximos permisibles (L.M.P) establecidos por la NOM-052-ECOL/1993. Las concentraciones por elemento, por método y por presa se reportan en el cuadro 4, el cual sólo presenta valores de concentración mínima, máxima, promedio y desviación estándar.

\section{Método NOM-053-ECOL-1993}

Los resultados obtenidos por esta prueba demostraron que la concentración de los metales considerados potencialmente tóxicos: $\mathrm{Cd}, \mathrm{Ni}, \mathrm{Ag}$ y As no rebasan el L.M.P. en las cuatro presas. En la figura 3 se reporta la concentración promedio obtenida para cada elemento en los extractos para cada presa de jales. En el análisis estadístico, la concentración de metales traza en muestras de sólidos (jales) mostraron una asociación geoquímica entre $\mathrm{Cd}$ y Zn. Esto se confirmó por medio de una gráfica de correlación de la concentración de los lixiviados entre ambos elementos, donde no sólo existe esta alta correlación entre las matrices, sino también entre la movilidad de ambos elementos. Por otra parte, se determinó que la concentración $\mathrm{de} \mathrm{Pb}$ en el lixiviado es superior a su L.M.P. en las presas Omitlán y Sur II. La concentración de $\mathrm{Zn}$ en el lixiviado representa las más elevada concentración de los metales disueltos en los extractos (de 10 a $100 \mathrm{mg} / \mathrm{l}$ aproximadamente), aunque para este metal no existe un límite máximo permisible en la NOM vigente. Por lo tanto, los resultados obtenidos con este método señalan que 
Cuadro 4

Análisis químicos de los lixiviados recuperados de las pruebas de diagnóstico de movilidad por los métodos NOM-053-ECOL-1993 y

EPA-1312 de las muestras de las presas de jales Omitlán, Dos Carlos, Sur I y Sur II del distrito minero Pachuca - Real del Monte

\begin{tabular}{|c|c|c|c|c|c|c|c|c|c|c|}
\hline Muestra/mg/L & $\mathrm{Cd}$ & $\mathrm{Pb}$ & $\mathrm{Zn}$ & $\mathrm{Cu}$ & $\mathrm{Mn}$ & $\mathrm{Fe}$ & $\mathrm{Ni}$ & $\mathrm{Ag}$ & Co & As \\
\hline \multicolumn{11}{|c|}{ MÉTODO NOM-053-ECOL-1993 } \\
\hline \multicolumn{11}{|c|}{ Presa Dos Carlos } \\
\hline Mínimo & 0,100 & 0,100 & 2,310 & 0,100 & 10,000 & 0,060 & 0,100 & 0,020 & 0,070 & 0,010 \\
\hline Máximo & 0,640 & 3,270 & 29,600 & 1,750 & 45,650 & 0,860 & 0,160 & 0,070 & 0,110 & 0,014 \\
\hline Promedio & 0,223 & 0,489 & 15,594 & 0,399 & 29,331 & 0,680 & 0,064 & 0,018 & 0,022 & 0,005 \\
\hline Desv.Están. & 0,212 & 1,086 & 14,154 & 0,479 & 16,525 & 2,151 & 0,037 & 0,018 & 0,021 & 0,005 \\
\hline \multicolumn{11}{|c|}{ Presa Sur I } \\
\hline Mínimo & 0,420 & 0,450 & 30,000 & 0,540 & 80,000 & 0,120 & 0,100 & 0,020 & 0,110 & 0,001 \\
\hline Máximo & 0,810 & 6,100 & 61,000 & 1,940 & 95,000 & 0,250 & 0,300 & 0,050 & 0,290 & 0,010 \\
\hline Promedio & 0,571 & 3,304 & 43,375 & 0,975 & 79,375 & 0,254 & 0,156 & 0,036 & 0,174 & 0,001 \\
\hline Desv.Están. & 0,158 & 2,486 & 13,049 & 0,711 & 14,412 & 0,232 & 0,085 & 0,012 & 0,089 & 0,004 \\
\hline \multicolumn{11}{|c|}{ Presa II } \\
\hline Mínimo & 0,020 & 5,900 & 35,000 & 0,061 & 52,950 & 0,036 & 0,010 & 0,020 & 0,050 & 0,010 \\
\hline Máximo & 0,795 & 32,500 & 155,000 & 1,100 & 117,000 & 1,960 & 0,060 & 0,030 & 0,110 & 0,030 \\
\hline Promedio & 0,406 & 29,142 & 49,764 & 0,520 & 64,986 & 0,568 & 0,034 & 0,014 & 0,040 & 0,005 \\
\hline Desv.Están. & 0,185 & 24,580 & 36,962 & 0,346 & 34,912 & 0,721 & 0,057 & 0,004 & 0,064 & 0,008 \\
\hline \multicolumn{11}{|c|}{ Omitlán } \\
\hline Mínimo & 0,430 & 5,700 & 8,000 & 0,120 & 8,600 & 0,100 & 0,020 & 0,010 & 0,010 & 0,001 \\
\hline Máximo & 1,400 & 50,350 & 105,000 & 2,550 & 47,550 & 0,390 & 11,000 & 0,022 & 0,070 & 0,006 \\
\hline Promedio & 0,630 & 16,515 & 91,006 & 1,283 & 53,256 & 0,094 & 0,017 & 0,014 & 0,019 & 0,004 \\
\hline Desv.Están. & 0,405 & 14,583 & 78,542 & 1,352 & 46,436 & 0,117 & 0,020 & 0,005 & 0,015 & 0,006 \\
\hline \multicolumn{11}{|c|}{ MÉTODO EPA13-12 } \\
\hline \multicolumn{11}{|c|}{ Presa Dos Carlos } \\
\hline Mínimo & 0,010 & 0,010 & 0,070 & 0,010 & 0,030 & 0,050 & ND & ND & 0,010 & 0,000 \\
\hline Máximo & 0,120 & 0,120 & 11,500 & 0,540 & 51,300 & 0,250 & 0,210 & 0,030 & 0,130 & 0,000 \\
\hline Promedio & 0,048 & 0,029 & 3,669 & 0,179 & 16,299 & 0,186 & 0,055 & 0,018 & 0,048 & 0,000 \\
\hline Desv.Están. & 0,046 & 0,059 & 4,833 & 0,221 & 21,981 & 0,072 & 0,104 & 0,010 & 0,052 & 0,000 \\
\hline \multicolumn{11}{|c|}{ Presa Sur I } \\
\hline Mínimo & ND & ND & 0,090 & 0,010 & 0,180 & 0,020 & ND & ND & 0,030 & 0,000 \\
\hline Máximo & 0,400 & 0,090 & 60,000 & 0,130 & 97,000 & 0,070 & 0,250 & 0,020 & 0,240 & 0,010 \\
\hline Promedio & 0,136 & 0,026 & 16,000 & 0,035 & 34,070 & 0,044 & 0,075 & 0,009 & 0,100 & 0,001 \\
\hline Desv.Están. & 0,174 & 0,035 & 24,449 & 0,043 & 41,621 & 0,019 & 0,110 & 0,004 & 0,083 & 0,004 \\
\hline \multicolumn{11}{|c|}{ Presa II } \\
\hline Mínimo & ND & 0,050 & 0,050 & 0,010 & 0,040 & 0,010 & ND & ND & ND & 0,000 \\
\hline Máximo & 0,290 & 0,090 & 20,000 & 0,030 & 38,800 & 0,120 & 0,070 & 0,020 & 0,110 & 0,000 \\
\hline Promedio & 0,063 & 0,042 & 3,693 & 0,007 & 8,346 & 0,037 & 0,010 & 0,001 & 0,018 & 0,000 \\
\hline Desv.Están. & 0,110 & 0,036 & 6,780 & 0,005 & 13,686 & 0,037 & 0,000 & 0,000 & 0,049 & 0,000 \\
\hline
\end{tabular}




\begin{tabular}{cccccccccccc}
\hline Muestra/mg/L & $\mathrm{Cd}$ & $\mathrm{Pb}$ & $\mathrm{Zn}$ & $\mathrm{Cu}$ & $\mathrm{Mn}$ & $\mathrm{Fe}$ & $\mathrm{Ni}$ & $\mathrm{Ag}$ & $\mathrm{Co}$ & $\mathrm{As}$ \\
\hline \multicolumn{10}{c}{ MÉTODO EPA13-12 } \\
Mínimo & $\mathrm{ND}$ & 0,060 & 0,040 & 0,010 & 0,120 & 0,020 & $\mathrm{ND}$ & $\mathrm{ND}$ & $\mathrm{ND}$ & 0,000 \\
Máximo & 0,050 & 2,810 & 9,000 & 0,140 & 7,650 & 0,590 & 0,010 & 0,020 & 0,020 & 0,000 \\
Promedio & 0,017 & 0,518 & 2,179 & 0,033 & 3,608 & 0,090 & 0,002 & 0,002 & 0,003 & 0,000 \\
Desv.Están. & 0,015 & 1,266 & 3,279 & 0,061 & 5,164 & 0,174 & 0,000 & 0,000 & 0,007 & 0,000 \\
\hline
\end{tabular}

los residuos de la presas Omitlán y Sur II podrían ser clasificados como residuos potencialmente peligrosos en función del plomo. Es importante subrayar que estos resultados podrían interpretarse como significativos, puesto que podrían representar la movilidad de los elementos analizados si los jales son dispersados (por viento, agua o transporte antropógeno) y depositados en suelos agrícolas. Bajo esta última condición, los minerales con contenidos metálicos si estarían expuestos a una solución extractante con ácidos orgánicos semejante a la utilizada en la prueba tipo NOM053-ECOL-1993.

\section{Método EPA-1312}

Con respecto a la calidad química de los lixiviados obtenidos por la prueba EPA-1312 de los metales potencialmente tóxicos (Cuadro 4) y para los cinco metales seleccionados por su toxicidad ( $\mathrm{Cd}, \mathrm{Pb}, \mathrm{Mn}, \mathrm{Zn}, \mathrm{As}$ ), se determinó que estos metales no rebasan el LMP en ninguna de las cuatro presas del distrito en base a sus valores promedio. Lo más significativo en los resultados de esta prueba es la concentración de $\mathrm{Mn}$ y $\mathrm{Zn}$ en las presas Omitlán, Sur I y Sur II, aunque no se cuenta con un LMP para estos metales, solo se observa una mayor concentración de $\mathrm{Pb}$ en la presa Omitlán, sin rebasar su LMP (Fig. 4). En el caso de los lixiviados de esta prueba no se pudo determinar si existe alguna correlación entre los elementos solubles en agua debido a que su concentración para algunos elementos químicos es menor al límite de detección del elemento por la técnica analítica empleada. Es importante señalar que la concentración de los metales traza disueltos con este agente extractante es significativamente menor en comparación a las concentraciones obtenidas para la prueba NOM-053. Sin embargo, la movilidad

\section{Concentración de metales tóxicos en lixiviados (NOM-053)}

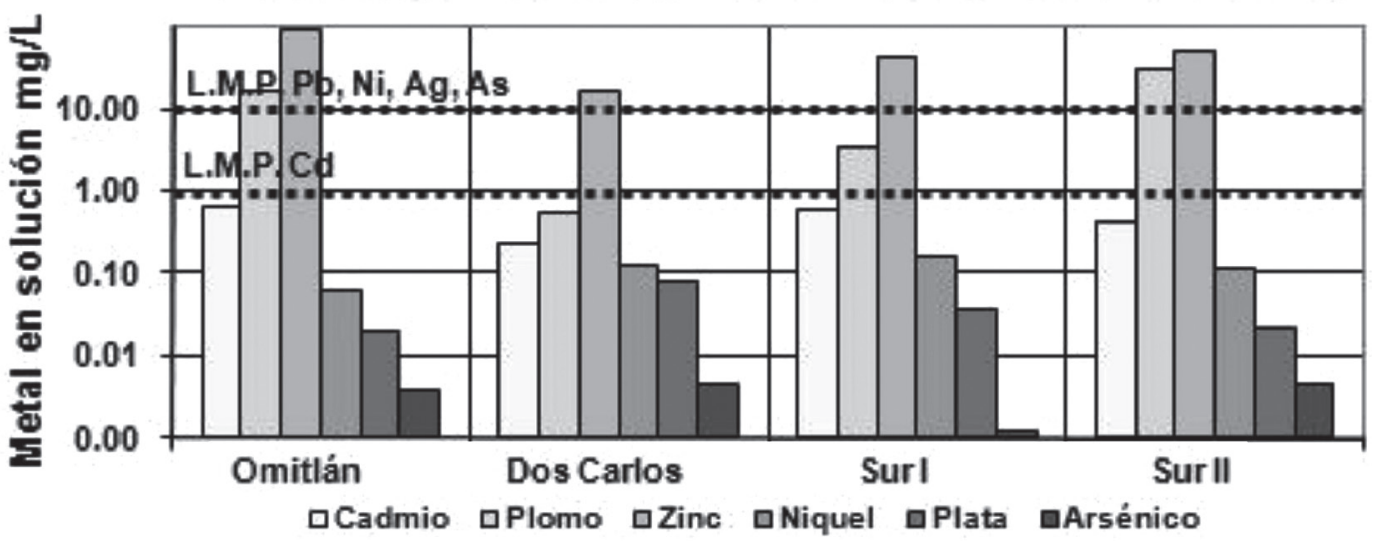

Fig. 3: Calidad química promedio de lixiviados recuperados de pruebas por el método NOM-053-ECOL-1993, de las muestras de las presas de jales del distrito minero Pachuca - Real del Monte. 


\section{Concentración de metales tóxicos en lixiviados (EPA-1312)}

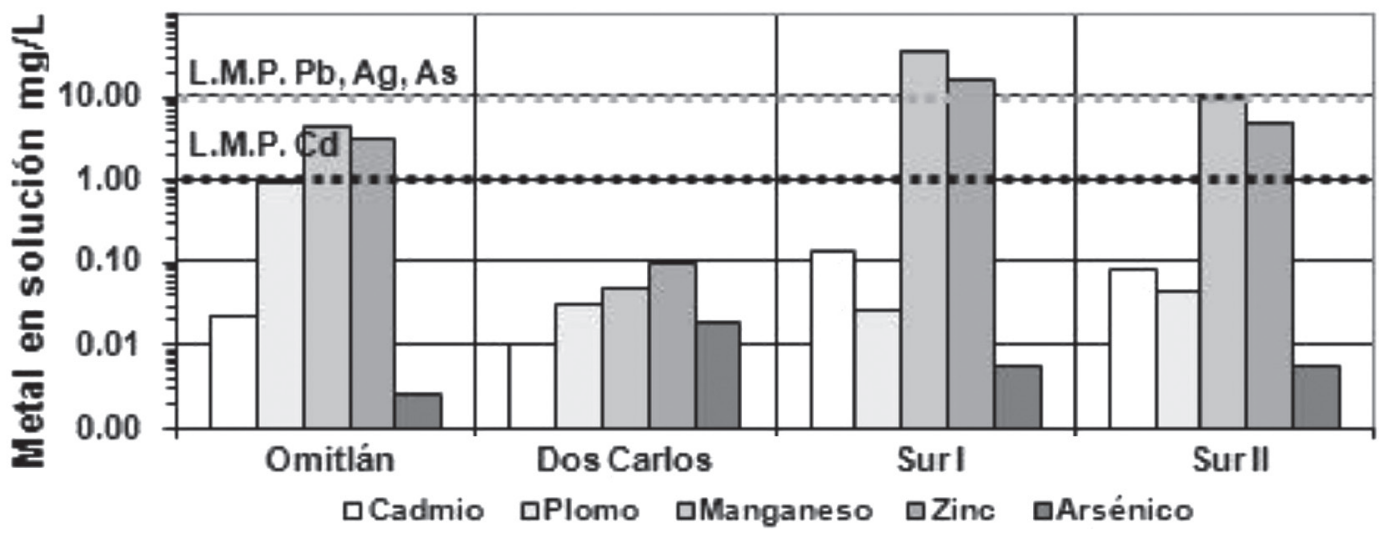

Fig. 4: Calidad química promedio de lixiviados recuperados de pruebas por el método EPA-1312 de las muestras de las presas de jales del distrito minero Pachuca - Real del Monte.

de los EPTs con una solución tipo EPA-1312 puede considerarse como más representativa de la exposición de los residuos (jales) al agua meteórica tal como sucede en las presas de jales.

\section{CONCLUSIONES}

Los jales provenientes de este tipo de yacimiento presentan concentraciones de elementos químicos considerados como potencialmente tóxicos entre los cuales destaca el $\mathrm{Pb}$ y el $\mathrm{Mn}$. La mineralización metálica es escasa, se observó que la pirita es el mineral más abundante seguida por goethita-hematita-lepidocrocita (fase mineral de neoformación que presenta la pirita), además de escasos sulfuros de $\mathrm{Zn}, \mathrm{Pb}, \mathrm{Cu}$ y $\mathrm{Fe}$ (esfalerita, galena, calcopirita) asociados a cuarzo, calcita y silicatos. La fase mineral mayoritaria determinada por difracción de rayos-X fue el cuarzo, lo que demuestra la naturaleza metalogenética del yacimiento (vetas de matriz cuarzosa) y la composición de la roca encajonante (rocas de composición ácida a intermedia). El análisis estadístico mostró la constante asociación bi-elemental de $\mathrm{Zn}$-Cd que se explica como la presencia de $\mathrm{Cd}$ en la estructura de la esfalerita ( $\mathrm{Zn}, \mathrm{Cd}, \mathrm{Fe}, \mathrm{Mn}) \mathrm{S}$ y/o como inclusión de greenockita (CdS). Aunque esta especie mineral es muy escasa en los residuos (jales). En el análisis factorial multielemental se aprecia la constante asociación $\mathrm{Cu}-\mathrm{Cd}-\mathrm{Zn}$ (esfalerita-calcopirita) y otras como Ba-Cr-Ni-Co atribuidos a impurezas dentro de la pirita y hematita-magnetita. Geoquímicamente se puede interpretar que estas concentraciones representan la mineralogía primaria relicta del yacimiento (principalmente sulfuros) la cual está expuesta a los procesos físicos y químicos de intemperismo. Así como a la neoformación de especies minerales como: óxidos e hidróxidos de $\mathrm{Fe}$ y $\mathrm{Cu}$ (goethita-lepidocrocitahematita, calcantita(?) y sulfatos (anglesita, yeso, jarosita, melanterita, entre otros). De manera general, los microanálisis del microscopio electrónico de barrido y la microsonda electrónica, mostraron que la principal neoformación mineralógica corresponde a los efectos de la alteración progresiva en la pirita y/o a la oxidación ferríca y precipitación de minerales sulfurosos que indican la presencia de los óxidos e hidróxidos de hierro y sulfatos. La prueba de diagnóstico de movilidad por el método NOM-053-ECOL-1993 mostró que la concentración de los elementos $\mathrm{Cd}$, $\mathrm{Ni}, \mathrm{Ag}$ y As, no rebasan el límite máximo permisible establecido por la NOM-052-ECOL-1993 en las cuatro presas del distrito. Aunque la concentración de $\mathrm{Pb}$ soluble es superior al L.M.P. en las presas Omitlán y Sur II. En cuanto al segundo método utilizado EPA-1312 se determinó que las concentraciones en solución de $\mathrm{Cd}, \mathrm{Pb}, \mathrm{Mn}, \mathrm{Zn}$ y As tampoco rebasan el límite máximo permisible 
señalado por la NOM-052-ECOL-1993 en ninguna de las presas. Lo más significativo es la concentración de Mn y Zn en las presas Omitlán, Sur I y Sur II y de Pb en Omitlán pero sin rebasar el límite máximo permisible. Comparando la movilidad para ambos tipos de soluciones extractantes se observó que la movilidad es menor en presencia de la solución tipo agua meteórica simulada. En el caso del plomo se diagnosticó para la solución tipo NOM-053-ECOL-1993 una mayor movilidad en las presas Omitlán y Sur II (concentración mayor de $10000 \mu \mathrm{g} . \mathrm{L}-1)$ lo que supone que estos residuos podrían considerarse potenciales fuentes de contaminación del suelo, sedimento y agua por $\mathrm{Pb}$. También, destaca la movilidad del $\mathrm{Zn}$ en la presa Sur I que se observa directamente asociado a la movilidad del Cd (presencia de Cd en la estructura de la esfalerita). Por último, el Mn sólo presenta movilidad significativa en la presa Sur I, aunque en los análisis químicos de sólidos (jales) se obtuvieron concentraciones significativas en la presa Omitlán. Además, la concentración de este elemento en el lixiviado, supone que este residuo puede ser considerado como fuente potencial de contaminación por Mn. A partir de estos resultados se puede hacer una síntesis de las fases minerales (primarias y secundarias) portadoras de los elementos considerados como potencialmente tóxicos en el distrito minero Pachuca - Real del Monte (Cuadro 5). En este cuadro resalta que no fue posible identificar, por las técnicas analíticas empleadas, alguna fase de neoformación portadora de algún elemento con mayor significado ambiental. Incluso, a través del microanálisis de las fases de neoformación de la pirita [goethitahematita-lepidocrocita, melanterita(?), mallardita(?)] no se identificó la presencia de algunos de los elementos potencialmente tóxicos (EPTs) identificados por microsonda electrónica en la pirita primaria (As, $\mathrm{Co}, \mathrm{Ni}$ y $\mathrm{Cr}$ ). Esto podría significar que estos elementos pudieron haber sido movilizados durante el proceso de alteración de la pirita o bien que se encuentran efectivamente asociados a los compuestos secundarios de hierro, pero por debajo del límite de detección de la microsonda electrónica.

Cabe señalar que la pirita siendo el mineral más abundante en estos jales, es el que presenta
Cuadro 5

Minerales primarios portadores de elementos potencialmente tóxicos (EPTs) en los jales del distrito minero Pachuca-Real del Monte

\begin{tabular}{cc}
\hline EPTs & Mineral primario \\
\hline $\mathrm{Pb}$ & Galena $(\mathrm{PbS})$ \\
$\mathrm{Cd}$ & Esfalerita $(\mathrm{Zn}, \mathrm{Cd}, \mathrm{Fe}, \mathrm{Mn}) \mathrm{S}$ \\
$\mathrm{As}$ & Arsenopirita $(\mathrm{FeAsS})$ \\
Pirita $(\mathrm{Fe}, \mathrm{As}, \mathrm{Cr}, \mathrm{Ni}, \mathrm{Co}) \mathrm{S}_{2}$ & \\
Proustita $\left(\mathrm{Ag}_{3} \mathrm{AsS}_{3}\right)$ & \\
$\mathrm{Cr}$ & Pirita $(\mathrm{Fe}, \mathrm{As}, \mathrm{Cr}, \mathrm{Ni}, \mathrm{Co}) \mathrm{S}_{2}$ \\
$\mathrm{Ni}$ & Pirita $(\mathrm{Fe}, \mathrm{As}, \mathrm{Cr}, \mathrm{Ni}, \mathrm{Co}) \mathrm{S}_{2}$ \\
$\mathrm{Co}$ & Pirita $(\mathrm{Fe}, \mathrm{As}, \mathrm{Cr}, \mathrm{Ni}, \mathrm{Co}) \mathrm{S}_{2}$ \\
$\mathrm{Ag}$ & Argentita $\left(\mathrm{Ag} \mathrm{S}_{2}\right)$ \\
& \\
\hline
\end{tabular}

mayor variedad en el grado de alteración. Esto puede estar relacionado a diferentes etapas en la historia de depósito: a).- deformación y alteración de la mineralización durante la formación del yacimiento; b).- explotación y c).- depósito de residuos. Evidencias de ello serían para una etapa, los huecos totalmente lixiviados tipo boxworks (Ramdohr, 1981). Otra etapa estaría representada por la pirita mejor preservada pero con fracturamiento perpendicular sobre sus planos, producto posiblemente de un esfuerzo tectónico. Y otra correspondería a la mineralización de pirita con bordes de alteración muy intensos provocados por la acción de los procesos de meteorización (principalmente hidrólisis) en las presas de jales. Por el porcentaje de pirita en la composición de estos jales, se espera que la formación de drenaje ácido sea a baja escala, sin embargo en áreas pequeñas de las presas se ha observado este efecto, manifestado por la formación de costras oxidadas muy superficiales.

\section{AGRADECIMIENTOS}

A la Universidad Autónoma del Estado de Hidalgo (UAEH) a través de sus programas de superación personal (PROMEP) y al Consejo Nacional de Ciencia y Tecnología (CONACyT) por el apoyo brindado para la realización de estudios de posgrado. 


\section{REFERENCIAS}

ALPERS, C.N., BLOWES, D.W., NORDSTROM, D.K. \& JAMBOR, J.L., 1994: Secondary minerals and acid mine-water chemistry.En: JAMBOR, J. L. \& BLOWES, D. W. (eds.). Short course handbook on environmental geochemistry of sulfide minewaste.- Mineral. Asoc. Canada. Nepean, 22: $247-270$.

ARAGÓN, A., TORRES, G., MONROY, M., LUSZCZEWSKI, A. \& LEYVA, R., 2000: Scanning electrón microscope and statistical análysis of suspended heavy metal particules in San Luis Potosí, Mexico.- Atmospheric Environment, 34: 4103-4112.

BONHAM, H.F.Jr., 1988: Bulk mineable gold deposits of the western United States.En KEAYS, R. R.; RAMSAY, W.R.H. \& GROVES, D.I. (eds): The geology of gold deposits. The perspective in 1988. Economic Geol., Monographs. 6: 193-207.

BLACK, R.F., 1963: Paragenesis.- En: GEYNE, A.R., FRIES, C.JR., SEGERSTROM, K., BLACK, R.F., \& WILSON, I.F., 1963: Geología y yacimientos minerales del distrito de Pachuca-Real del Monte, Estado de Hidalgo, México: México, D. F.- Consejo de Recursos Naturales no Renovables, Pub. 5E: 222.

BLOWES, D.W. \& PTACEK, C.J., 1994: Acidneutralization mechanisms in inactive mine tailings.- En: JAMBOR, J.L. \& BLOWES, D.W. (eds). Short course handbook on environmental geochemistry of sulfide minewaste.- Mineral. Asoc. Canada. Nepean, v. 22: 271-291.

CALDWELL, J.A. \& WELSH, J.D., 1982: Tailings disposal in ruggeg, hig precipitation environments: an overview and comparative assessment.- En: ELLIS, D.V., (eds): Marine tailings disposal.- Ann. Arbor Science: 5-62.
CASTAÑEDA, E.P., 2002: Experimentos de laboratorio para evaluar los solutos lixiviables en jales del distrito minero Pachuca-Real del Monte.- 61 págs. Univ. Nacional Autónoma de México, México, [Tesis M.Sc.].

DAMON, P.E., SHAFIQULLAH, M. \& CLARK, K.F., 1981: Evolución de los arcos magmáticos en México y su relación con la metalogénesis. Instituto de Geología, Universidad Nacional Autónoma de México.- Revista Mexicana de Ciencias Geológicas, 5: 223-238.

DANIELSSON, A., CATO, I., CARMAN, R. \& RAHM, L., 1999: Spatial clustering of metals in the sediments of the Skagerrak/Kattegat.Applied Geochemistry, 14: 689-706.

DRIER, J.E., 1976: The geochemical environment of ore deposition in the Pahuca-Real del Monte district, Hidalgo, Mexico.- 115 págs. Univ. de Arizona, Tucson Az., [Tesis PhD.].

FACCHINELLI, A., SACCHI, E. \& MALLEN, L., 2001: Multivariate statical and GISbased approach to identify heavy metal sources in soils.- Environmental Pollution, 114: 313-324.

FREEZE, R. \& CHERRY, J.A., 1979: Groundwater.- 604 págs. Ed. Prentice-Hall. EngleWood Cliffs, New Jersey.

GEYNE, A.R., FRIES, C.JR., SEGERSTROM, K., BLACK, R.F. \& WILSON, I.F., 1963: Geología y yacimientos minerales del distrito de PachucaReal del Monte, Estado de Hidalgo, México: México, D. F.- Consejo de Recursos Naturales no Renovables, Pub. 5E: 222.

HAYBA, D.O., BETTHKE, P.M., HEALD, P. \& FOLEY, N.K., 1985: Geologic, mineralogic and geochemical characteristics of volcanic-hosted epithermal precious-metal deposits.- En: BERGER, B.R. \& BETHKE, P.M., (eds): Geology and geochemistry of epithermal systems.- Economic Geol., Reviews, 2: 129-168. 
HEALD, P., FOLEY, N.K. \& HAYBA, D.O., 1987: Comparative anatomy of volcanichosted epithermal deposits-Acid-sulfate and adularia-sericite types.- Economic Geol., 82: 1-26.

JAMBOR, J.L., 1994: Mineralogy of sulfide-rich tailings and their oxidation products.- En: JAMBOR, J.L. \& BLOWES, D.W. (eds). Short course handbook on environmental geochemistry of sulfide mine-waste.- Mineral. Asoc. Canada. Nepean, 22: 247-270.

KELLY, E.G. \& SPOTTISWOD, D.J., 1990: Disposición de las colas.- En: KELLY, E.G. Introducción al procesamiento de minerales. Ed. Limusa: 423-427.

KLEIN, C., 2002: Manual of Mineral Science $\left[22^{\text {nd }}\right.$ ed].- 641 págs. John Wile \& Sons, London.

MORENO R., 1998: Caracterización mineralógica y química de desechos mineros (jales), aplicada a la recuperación de valores económicos en Zimapán y Pachuca, Edo. de Hidalgo.- 89 págs. Inst. Politécnico Nacional, México [Tesis M.Sc.].

NORMA OFICIAL MEXICANA, NOM-052ECOL-1993: Establece los criterios para definir las características de los residuos clasificados como residuos peligrosos. Diario Oficial de la Federación Mexicana.

NORMA OFICIAL MEXICANA, NOM-053ECOL-1993: Procedimiento para llevar a cabo la prueba de extracción para determinar los constituyentes que hacen a un residuo peligroso por su toxicidad al ambiente. Diario Oficial de la Federación Mexicana.
RAMDOHR, P., 1981: The ore minerals and their intergrowths. International series in Earth Sciences,- 1174 págs. Pergamon Press, 2nd. Edition.

REIMANN, C., FILZMOSER, P. \& GARRET, G.R., 2002: Factor analysis applied to regional geochemical data: problems and possibilities.- Applied Geochemistry, 17: 185-206.

SILBERMAN, M.L. \& BERGER, B.R., 1985: Relationship of trace-elements patterns to alteration and morphology in epithermal precious-metal deposits.En BERGER, B.R. \& BETHKE, P.M., (eds.): Geology and geochemistry of epithermal systems. - Economic Geol., Reviews, v. 2: 129-168.

SMITH, S.K. \& HUYCK, O.L.H., 1999: An overview of the abundance, relative mobility, bioavailability, and human toxicity of metales. The Environmental Geochemistry of Mineral Deposits. Part A: Processes, Techniques, and Health Issues.- Economic Geol., Reviews 6A: 29-70.

YTA, M., 1992: Étude géodynamique et metallogénique d'un secteur de la Faja de Plata, Mexique : La zone de Zacatecas-Francisco I. Madero.- 266 págs. Univ. d'Orleans, Francia [Tesis $\mathrm{PhD}$.].

YTA, M. \& MORENO R., 1997: La mineralización en los distritos mineros PachucaReal del Monte y Zimapán. Su papel en la evolución metalogénica del estado de Hidalgo, México.- II Convención sobre la evolución geológica de México y recursos asociados. Guía de excursiones: 73-84. 
